ARTICULO

Revista Derecho - Año 2 edición 3: 247 - 259

Web: http://www.revistaderecho.pe E-mail: editorial@revistaderecho.pe

ISSN 2313-6944

\title{
ANÁLISIS DE LA IDONEIDAD DE LAS MEDIDAS DE PROTECCIÓN DICTADAS A FAVOR DE LAS VÍCTIMAS DE VIOLENCIA EN EL MARCO DE LA LEY 30364 "LEY PARA PREVENIR, SANCIONAR Y ERRADICAR LA VIOLENCIA CONTRA LAS MUJERES Y LOS INTEGRANTES DEL GRUPO FAMILIAR
}

Pamela Yhosely Calisaya Yapuchura

INFORMACIÓN DEL ARTICULO

Art. Recibido: 04/02/16 Art. Aceptado: 01/06/16

Art. Publicado: $18 / 12 / 18$

PALABRAS CLAVE:

Medidas de protección

Violencia

Violencia de género Violencia contra los integrantes del grupo familiar Idoneidad

\section{RESUMEN}

El presente trabajo consiste en el análisis de las medidas de protección dictadas a favor de las víctimas de violencia en el marco de la Ley 30364, "Ley para prevenir, sancionar y erradicar las violencia contra las Mujeres y los Integrantes del grupo familiar”. El propósito es determinar si estas medidas de protección dictadas a favor de las víctimas de violencia son idóneas o no, siendo la pregunta a responder ¿¿Son idóneas las medidas de protección dictadas a favor de las víctimas de violencia, dentro del marco de la Ley 30364? Y arribando a las siguiente conclusion : i) Pese a que las medidas de protección son urgentes, preventivas y no determinan la responsabilidad del denunciado, su vigencia y mantenimiento depende de la decisión final que dicte la Fiscalía Penal o Juzgado de Paz Letrado, lo que acarrea como consecuencia que las mismas no sean idóneas.

\section{“ANALYSIS OF THE SUITABILITY OF PROTECTIVE MEASURES \\ DICTATED IN FAVOR OF VICTIMS OF VIOLENCE IN THE FRAMEWORK \\ OF LAW 30364" AN ACT TO PREVENT, PUNISH AND ERADICATE VIOLENCE AGAINST WOMEN AND MEMBERS OF THE FAMILY GROUP"}

ARTICLE INFO

Article Received: 04/02/16

Article Accepted: 01/06/16

Article Published: 18/12/18

\section{ABSTRACT}

The present work consists in the analysis of the protection measures issued in favor of the victims of violence within the framework of Law 30364, "Law to prevent, punish and eradicate violence against Women and Family Members".

Abogada titulada en la Universidad Nacional del Altiplano. Correo electrónico: pamelayhosely_cy@hotmail.com 
KEY WORDS:

Protection measures

Violence

Gender violence

Violence against members of the

family group

Suitability
The purpose is to determine if these protective measures issued in favor of victims of violence are suitable or not, and the question to be answered are the measures of protection dictated in favor of victims of violence, within the framework of Law 30364. ? And arriving at the following conclusion: i) Although the protection measures are urgent, preventive and do not determine the respondent's responsibility, its validity and maintenance depends on the final decision issued by the Criminal Prosecutor's Office or Magistrate's Court of Justice, which As a consequence, they are not suitable. 


\section{INTRODUCCIÓN}

El presente artículo se centra en responder la siguiente interrogante: ¿Son idóneas las medidas de protección dictadas a favor de las víctimas de violencia en el marco de la Ley 30364 ? Y para ello, es necesario desarrollar y analizar ciertos aspectos, tales como: determinar el concepto de medidas de protección, el concepto de idoneidad, y el concepto de medidas autosatisfactivas; conceptos que nos permitirá responder la interrogante antes planteada. Asimismo, la necesidad de definir tales conceptos se da a raíz de que la Ley 30364, no desarrolla de forma clara dichos conceptos, los mismos que son necesarios para entender los alcances de las medidas de protección.

Si bien, se cree que el tema de la violencia familiar es un tema recurrente, vano y sin ninguna relevancia jurídica, tal acepción está muy lejos de la realidad, ya que la violencia se ha convertido en un problema anacrónico que se encuentra acuñada en nuestra sociedad, y la misma (OMS, 2013) la identifica como una pandemia que afecta al $50 \%$ de la población mundial, habiendo sido hasta un $70 \%$ de las mujeres víctimas de violencia en algún momento de su vida. Estos estudios destacan la necesidad de que todos los sectores se comprometan en eliminar la tolerancia de la violencia contra las mujeres, varones, niños, personas de la tercera edad, personas discapacitadas y demás víctimas de violencia, por lo que su pronta solución resulta ser de importancia no solo nacional, sino más bien internacional, y si bien, a lo largo del tiempo se han dictado medidas para proteger a las víctimas, tales medidas no han sido suficientes e idóneas, pues las mismas no se han manifestado en cifras ni en la disminución de casos de violencia.

Frente a estas consideraciones, las autoridades estatales peruanas emprendieron una serie de iniciativas legislativas para tratar esta problemática, cabe mencionar que el Perú en América latina fue uno de los primeros países que ha adoptado una Ley especial sobre violencia familiar, ley 26260, ley de protección frente a la violencia familiar, publicada en diciembre de 1993, y reforzada posteriormente en 1997, 2000 y finalmente el 27 de julio de 2010, con el Decreto Supremo No 006-97-JUS, Texto Único Ordenado de la Ley $\mathrm{N}^{\circ}$ 20260 "Ley de Protección frente a la Violencia Familiar"; esta ley se caracterizaba por seguir el trámite de un proceso ordinario, es decir, el proceso se iniciaba interponiendo la demanda ya sea de parte (victima) o del representante del Ministerio público, se corría traslado a la parte demandada por el plazo de cinco días de notificado para absolver la demanda, se realizaba una audiencia única, y se emitía una sentencia donde se resolvía: i) si ha existido violencia familiar o no, ii) se dictaba medidas de protección, iii) el tratamiento psicológico a la víctima, iv) y la reparación del daño; proceso que duraba aproximadamente seis meses, dejando en situación de vulnerabilidad a las víctimas de violencia mientras esperaban la emisión de las sentencias para obtener me- 
didas de protección; la ley daba un alcance de plan de políticas públicas que manifestaban al menos un ideal político: la protección de las víctimas y la erradicación de la violencia familiar en nuestro país, sin embargo tal protección no se manifestó en cifras, pues los casos de violencia familiar en los Juzgados de Familia iban en aumento y las víctimas de las mismas seguían desprotegidas. Por ello, el 23 de noviembre de 2015 se dictó la Ley N³0364, "Ley para prevenir, sancionar y erradicar la violencia contra la mujer y los integrantes del grupo familiar" con el objetivo de prevenir, erradicar y sancionar toda forma de violencia producida en el ámbito público o privado contra las mujeres por su condición de tales, y contra los integrantes del grupo familiar; en especial, cuando se encuentran en situación de vulnerabilidad, por la edad o situación física como las niñas, niños, adolescentes, personas adultas mayores y personas con discapacidad. Y el 27 de julio de 2016 se publicó el Decreto Supremo No 009-2016MIMP, que aprueba el Reglamento de la Ley No 30364, Ley para prevenir, sancionar y erradicar la violencia contra las mujeres y los integrantes del grupo familiar. La presente ley y su Reglamento se caracterizan por la inmediatez en su tramitación y por centrarse en tres etapas: prevención de la violencia, protección de las víctimas y sanción al agresor, enfocándose el presente artículo en la etapa de protección de las víctimas de violencia, y específicamente en la idoneidad de las medidas de protección.

\section{LA VIOLENCIA}

Previamente a desarrollar el concepto de medidas de protección, es necesario desarrollar algunos conceptos básicos, siendo el más importante, el concepto de violencia.

El maestro Guillermo Cabanellas señala que por violencia se entiende aquella "situación o estado contrario a la naturaleza, modo o índole" consistente en el "empleo de la fuerza para arrancar el consentimiento" (CABANELLAS, 2003).

Jorge Corsi, destacado psicólogo e investigador del tema de violencia familiar refiere que "la raíz etimológica del término violencia remite al concepto de fuerza. El sustento violencia se corresponde con verbos tales como violentar, violar, forzar" (CORSI, 2009). Siguiendo el hilo argumentativo de Corsi "para que la conducta violenta sea posible, tiene que darse una condición: la existencia de un cierto desequilibrio de poder, que puede ser definida culturalmente, por el contexto $\mathrm{u}$ obtenido a través de maniobras interpersonales de control de relación.

Asimismo, resulta interesante el aporte de Tornes Falcón quien nos dice que la violencia es el acto que produce daños de índole y magnitud diversas, transgrede el derecho de la víctima, su integridad física, emocional y sexual. (VILLANUEVA, 2003).

La OMS (OMS, 2002) define la violencia como "el uso deliberado de la fuerza física o el poder, ya sea en grado de amenaza o efectiva, contra 
uno mismo, otra persona o un grupo o comunidad, que cause o tenga muchas probabilidades de causar lesiones, muerte, daños psicológicos, trastornos del desarrollo o privaciones".

\section{VIOLENCIA CONTRA LA MUJER}

La violencia de género se manifiesta "a través de conductas y actitudes basadas en un sistema de creencias sexista y heterocentrista, que tienden a acentuar las diferencias apoyadas en estereotipos de género, conservando las estructuras de dominio que se derivan de ellos. La violencia de género adopta formas muy variadas, tanto en el ámbito de lo público, como en los contextos privados. Ejemplos de ella son, entre otras, todas las formas de discriminación hacia la mujer en distintos niveles (político, institucional, laboral), el acoso sexual, la violación, el tráfico de mujeres para la prostitución, la utilización del cuerpo femenino como objeto de consumo, la segregación basada en ideas religiosas y, por supuesto, todas las formas de maltrato físico, psicológico, social, sexual que sufren las mujeres en cualquier contexto, y, que ocasionan una escala de daños que puedan culminar en la muerte (MOLINA, 2010).

Las Naciones Unidas (OMS, 2013) define la violencia contra las mujeres como "Todo acto de violencia de género que resulte, o pueda tener como resultado un daño físico, sexual o psicológico para la mujer, inclusive las amenazas de tales actos, la coacción o la privación arbitraria de libertad, tanto si se producen en la vida pública como en la privada", reconociéndolo como una pandemia que afecta al $50 \%$ de la población mundial, habiendo sido hasta un $70 \%$ de las mujeres víctimas de esa violencia en algún momento de su vida(OMS, 2013).

Para la Cumbre Judicial Iberoamericana(IBOAMERICA, 2008) se considera violencia contra la mujer cualquier acción o conducta, basada en su género, que cause muerte, daño o sufrimiento físico, sexual o psicológico a la mujer, tanto en el ámbito público como en el privado, mediante el empleo de la violencia física o psíquica.

\section{VIOLENCIA CONTRA LOS INTEGRANTES DEL GRUPO FAMILIAR}

La violencia doméstica “(....) alude a todas las formas de abuso que tiene lugar en las relaciones entre quienes sostienen o han sostenido un vínculo afectivo relativamente estable". Se denomina relación de abuso a aquella "forma de interacción que, enmarcada en un contexto de desequilibrio de poder, incluye conductas de una de las partes que, por acción o por omisión, ocasionan daño físico y/o psicológico a otro miembro de la relación" (CORSI, 2007)

Asimismo como (CORSI, 2007) señala la violencia está constituida por el abuso de poder a los grupos más vulnerables, más débiles, identificando en esta campo a los niños, niñas, mujeres y personas adultas. 
Asimismo, la Ley 30364 Ley para prevenir, sancionar y erradicar la violencia contra la mujer y los integrantes del grupo familiar en su artículo $6^{\circ}$ define que la violencia contra los integrantes del grupo familiar es cualquier acción o conducta que causa muerte, daño o sufrimiento físico, sexual o psicológico y que se produce en el contexto de una relación de responsabilidad, confianza o poder, de parte de un integrante a otro del grupo familiar.

\section{TIPOS DE VIOLENCIA}

\subsection{Maltrato fisico}

El maltrato físico es el acto (acción u omisión) de agresión, que de forma directa o indirecta, causa una afectación material en el cuerpo, salud o inactividad vital de la víctima. En este caso, el agresor tiene la intención de inferir un daño físico, siendo su fin inmediato ese, causar el daño, pero también el agresor de violencia familiar tiene un fin mediato, pues con su agresión lo que busca es someter o posicionarse como superior al agraviado (CRHISTIAN, 2009).

Tornes Falcón dice que la violencia física se manifiesta de manera latente, porque el daño producido se marca en el cuerpo de la víctima, como los golpes, heridas, mutilaciones, a veces producen lesiones internas que solo son identificables tras un periodo más o menos prolongado y que incluso, llegan a ocasionar la muerte (FALCON, 2006).

\subsection{El maltrato psicologico}

El maltrato psicológico implica la afectación emocional que sufre la victima ante la conducta violenta del agresor. Sea la forma en que se manifieste dicha conducta agresiva: física, verbal, sexual, económica, etc., la victima siempre padecerá del miedo, ansiedad, depresión, desesperación, inseguridad, desvalorización y demás afecciones emocionales propias del maltrato psicológico. (SALAS BETETA, 2009).

Asimismo, la violencia psicológica es la acción o conducta que puede ocasionar daños psíquicos, que consiste en la afectación o alteración de algunas funciones mentales. Daño psíquico es la afectación o alteración de algunas de las funciones mentales o capacidades de la persona, producida por un hecho o un conjunto de situaciones de violencia, que determina un menoscabo temporal o permanente, reversible o irreversible del funcionamiento integral previo. Así se desprende del literal b) del artículo 8 , de la Ley 30364.

\subsection{El maltrato sexual o violencia sexual}

El maltrato sexual es la "acción u omisión mediante la cual se induce o se impone la realización de prácticas sexuales no deseadas o respecto de las cuales se tiene incapacidad para consentir. Las relaciones sexuales forzadas en la pareja, también son consideradas violaciones y las victimas deben recibir la atención adecuada. El maltrato sexual también comprende la prohibi- 
ción del libre acceso y uso de métodos anticonceptivos y para la prevención de ITS.

Así también la Ley 30364, define la violencia sexual como acciones sexuales que se cometen contra una mujer o integrante del grupo familiar sin su consentimiento o coaccionándola, considerándose además como violencia sexual a la exposición de material pornográfico.

\subsection{Violencia economica o patri- monial}

La violencia económica o patrimonial es la acción u omisión que ocasiona daño o sufrimiento a través de menoscabar los recursos económicos o patrimoniales de las mujeres por su condición de tales o contra cualquier integrante del grupo familiar, en el marco de relaciones de poder, responsabilidad, confianza, en especial contra las niñas, niños, adultos mayores o personas con discapacidad.

De igual forma la violencia económica es la acción u omisión que se dirige a ocasionar un menoscabo económico a la víctima de violencia, según fluye del literal d) del artículo 8 de la Ley 30364.

\section{VICTIMA}

Es la mujer o integrante del grupo familiar que ha sufrido daño ocasionado por cualquier acción u omisión. Las niñas, niños y adolescentes, que hayan estado presentes en el momento de la comisión del delito, o que hayan sufrido daños por haber intervenido para prestar asistencia a la víctima o por cualquier otra circunstancia en el contexto de la violencia, son consideradas víctimas. Se incluye, además, de acuerdo al caso particular, a la familia del entorno inmediato o a las personas que están a cargo de la víctima.

A efectos de las 100 reglas de Brasilia (IBOAMERICA, 2008), se considera víctima toda persona física que ha sufrido un daño ocasionado por una infracción penal, incluida tanto la lesión física o psíquica, como el sufrimiento moral y el perjuicio económico. El término víctima también podrá incluir, en su caso, a la familia inmediata o a las personas que están a cargo de la víctima directa.

\section{PERSONAS EN SITUACIÓN DE VULNERABILIDAD}

Son las personas que por razón de su edad, género, estado físico o mental, o por circunstancias sociales, económicas, étnicas o culturales, se encuentren con especiales dificultades para ejercer con plenitud sus derechos reconocidos por el ordenamiento jurídico. Pueden constituir causas de vulnerabilidad, entre otras, las siguientes: la edad, la discapacidad, la pertenencia a comunidades indígenas o a minorías, la migración y el desplazamiento interno, la pobreza, el género, la orientación sexual y la privación de libertad.

Para la Cumbre Judicial Iberoamericana (IBOAMERICA, 2008) se consideran en condición de vulne- 
rabilidad aquellas personas que, por razón de su edad, género, estado físico o mental, o por circunstancias sociales, económicas, étnicas y/o culturales, encuentran especiales dificultades para ejercitar con plenitud ante el sistema de justicia los derechos reconocidos por el ordenamiento jurídico.

\section{LA DIGNIDAD DE LA PERSONA}

El artículo $1^{\circ}$ de la Declaración Universal de Derechos Humanos de Naciones Unidas del 10 de diciembre de 1948, determina que todos los seres humanos nacen libres e iguales en dignidad y derechos. Dotados de razón y de conciencia, debiendo actuar unos con los otros en un espíritu de fraternidad.

La dignidad de la persona es el rasgo distintivo de los seres humanos respecto de los demás seres vivos, la que constituye a la persona como un fin en sí mismo, impidiendo que sea considerada un instrumento o medio para otro fin, además de dotarlo de capacidad de autodeterminación y de realización del libre desarrollo de la personalidad. La dignidad es así un valor inherente a la persona humana que se manifiesta a través de la autodeterminación consciente y responsable de su vida y que exige el respeto de ella por los demás. (HUMBERTO, 2014)

Ingo Wolfgang Sarlet sostiene que la dignidad de la persona humana es una cualidad intrínseca y distintiva reconocida a todo individuo que lo hace merecedor del mismo respeto y consideración por parte del Estado y de la comunidad, implicando, en este sentido, un complejo de derechos y deberes fundamentales que aseguran a la persona tanto contra todo y cualquier acto de cuño degradante o deshumanizado, como velan por garantizar las condiciones existenciales mínimas para una vida saludable, además de propiciar y promover su participación activa y corresponsable en los destinos de la propia existencia y de la vida en comunión con los demás seres humanos, mediante el debido respeto a los demás seres que integran la red de la vida (Sarlet, 2009)

Por lo mismo, cada derecho reconocido en nuestra Constitución y leyes especiales protege el respeto a la dignidad de la persona, garantizan las condiciones mínimas para vivir una vida saludable, y haciendo referencia al tema del artículo, garantizan el derecho a vivir una vida sin violencia.

La Ley No 30364 "Ley para prevenir, sancionar y erradicar la violencia contra la mujer y los integrantes del grupo Familiar" establece como un derecho en su artículo 9, a el principal derecho que constituye materia de este trabajo

\section{Artículo 9. Derecho a una vida libre de violencia}

Las mujeres y los integrantes del grupo familiar tienen derecho a una vida libre de violencia, a ser valorados y educados, a estar libres de toda forma de discriminación, estigmatización y de patrones estereotipados de comportamientos, prácticas sociales y culturales 
basadas en conceptos de inferioridad $y$ subordinación.

Esta ley, remarca la importancia de vivir en una vida sin violencia, lo que implica que las personas- mujeres, varones, niños, niñas, adultos mayores- tienen derecho a disfrutar de un ambiente sano, sin agresiones de ningún tipo, estar libres de cualquier clase de discriminación y patrones estereotipados de comportamientos que los denigren como personas, este derecho es uno de los más importantes ya que resaltan la necesidad de las personas de vivir en un mundo donde son aceptados y respetados como son, lo que salvaguarda su dignidad humana.

Las 100 Reglas de Brasilia sobre acceso a la Justicia de las personas en Condición de Vulnerabilidad (IBOAMERICA, 2008), en su exposición de motivos refiere que el sistema judicial se debe configurar, y se está configurando como un instrumento para la defensa efectiva de los derechos de las personas en condición de vulnerabilidad, poca utilidad tiene que el estado reconozca formalmente un derecho si su titular no puede acceder de forma efectiva al sistema de justicia para obtener la titular de dicho derecho.

Si bien la dificultad de garantizar la eficacia de los derechos afecta con carácter general a todos los ámbitos, se deberá llevar a cabo una actuación más intensa para vencer, eliminar o mitigar dichas limitaciones. De esta manera el propio sistema de justicia puede contribuir de forma importante a la reducción de las desigualdades sociales, favoreciendo la cohesión social.

\section{MEDIDAS DE PROTECCIÓN}

Determinar el concepto de medidas de protección, es trascendental para los fines del presente artículo, ya que conocer los alcances que tiene la misma nos va a permitir entender su naturaleza e importancia.

La etapa de protección que regula la Ley 30364, se manifiesta a través de la emisión de las medidas de protección, por ello, su conceptualización debe ser clara y precisa.

El Reglamento de la Ley 30364, regula en su Art. $31^{\circ}$ las medidas de protección como "(...) el Juzgado de Familia dicta la medida de protección más idónea para el bienestar y seguridad de la víctima, atendiendo a las circunstancias particulares del caso, los resultados de la ficha de valoración del riesgo, la pre existencia de denuncias por hechos similares, la relación de la víctima con la persona denunciada, la diferencia de edades o relación de dependencia entre la víctima y la persona denunciada y, la situación económica y social de la víctima, entre otros aspectos que revelen vulnerabilidad (...).

Al respecto (DIAZ POME, 2009) refiere que “(...) Las medidas de protección son aquellas actitudes y decisiones que toma en cuenta el Estado a través de sus diversas instituciones públicas, a fin de hacer efectivo el cuidado y protección de la víctima de la agresión, con respecto a la agresión misma y a su agresor; son mecanismos que buscan brindar apoyo y protección a las víctimas de las agresiones e 
impedir la continuación de estas. Asimismo, estas medidas de protección van más allá, por cuanto buscan que la víctima se sienta tranquila y que pueda gradualmente volver a su vida normal, rehabilitándola de sus traumas. Dichas medidas de protección se encuentran establecidas en nuestra legislación (...)”.

En consecuencia, las medidas de protección son aquellas decisiones que el Juez de Familia toma para cuidar de manera preventiva a la víctima de violencia frente a un eventual riesgo de sufrir un nuevo acto de violencia por causa del agresor, con el fin de salvaguardar su dignidad.

\section{CONCEPTO DE IDÓNEO}

Para Guillermo Cabanillas de Torres, la idoneidad significa "Con aptitud legal para ciertos actos; como servir de testigo, por no estar incurso en ninguna de las incapacidades por la ley previstas".(CABANELLAS DE TORRES, 2011)

Para la Enciclopedia Jurídica (JURIDICA, 2014) "En derecho, se habla de idoneidad para referirse a algo o alguien que es capaz o que reúne las condiciones suficientes para desempeñar una función dentro de un proceso. Así por ejemplo se considerara la idoneidad del testimonio de un perito con relación a una prueba específica en función a su conocimiento y preparación para emitir en determinada causa".

El adjetivo idóneo o idóneo se utiliza para expresar que algo resul- ta adecuado o conveniente para un propósito. En consecuencia, es todo aquello que posee la condición de idóneo.

Como tal, se refiere a la aptitud, buena disposición o capacidad que algo tiene o alguien tiene para un fin determinado. La palabra proviene del latinidoneitas, idoneitatis.

En otras palabras, para los fines del articulo la idoneidad se refiere a algo que reúne las condiciones suficientes para efectivizar un propósito, es decir, es el medio para alcanzar un fin.

11. MEDIDAS DE
PROTECCIÓN
AUTOSATISFACTIVAS
Peirano (PEIRANO, s.f.) con-
ceptualiza las medidas autosatisfactivas como soluciones jurisdiccionales urgentes, autónomas, "inaudita et altera pars" y mediando una fuerte probabilidad de que los planteamientos formulados sean atendibles. Importan una satisfacción definitiva de los requerimientos de sus postulantes y constituyen una especie de la tutela de urgencia que debe distinguirse de otras, como por ejemplo, de las diligencias cautelares clásicas. Pueden llegar a desempeñar un rol trascendental para remover "vías de hecho'; sin tener que recurrir al efecto a la postulación de diligencias cautelares que, como se sabe, ineludiblemente requieren la iniciación de una pretensión principal que, a veces, no desean promover los justiciables. 
Tiene las siguientes características:

a) Su despacho (el de la medida autosatisfactiva) reclama una fuerte probabilidad de que lo pretendido por el requirente sea atendible.

b) Su dictado acarrea una satisfacción "definitiva" de los requerimientos del postulante (salvo, claro está, que el destinatario de la precautoria hubiera articulado exitosamente las impugnaciones del caso),

c) Se genera un proceso (a raíz de la iniciación de una medida autosatisfactiva) que es autónomo en el sentido de que no es tributario ni accesorio respecto de otro, agotándose en sí mismo.

En síntesis las medidas autosatisfactivas son soluciones jurisdiccionales urgentes, autónomas. Las mismas importan una satisfacción definitiva de los requerimientos de los postulantes, motivos por el cual se sostiene que son autónomas, no dependiendo su vigencia y mantenimiento de la interposición coetánea o ulterior de una pretensión principal.

En suma, se concluye que son medidas de protección idóneas aquellas decisiones que el Juez de Familia dicta para proteger de manera preventiva a la víctima de violencia frente al eventual riesgo de sufrir un nuevo acto de violencia a causa de su agresor, esto atendiendo a las circunstancias particulares del caso en concreto, la ficha de valoración de riesgo, y demás circuns- tancias que demuestren la situación real de la víctima frente a su agresor, ponderando la proporcionalidad entre la eventual afectación causada y la medida de protección a adoptarse, dependiendo su vigencia y mantenimiento del tiempo necesario que necesite la victima de estas medidas.

Concepto que difiere con lo regulado en la Ley 30364, art. 23, en relación a la vigencia de las medidas de protección, la misma que establece que "(...) la vigencia de las medidas dictadas por el juzgado de familia o su equivalente se extiende hasta la sentencia emitida en el juzgado penal o hasta el pronunciamiento fiscal por el que se decida no presentar denuncia penal por resolución denegatoria, salvo que estos pronunciamientos sean impugnados (...)".

\section{CONCLUSIONES}

La Ley 30364 "Ley para prevenir, sancionar y erradicar la violencia contra las mujeres y los integrantes del grupo familiar" al supeditar la vigencia de las medidas de protección a la Sentencia final del Juez Penal o hasta el pronunciamiento fiscal, le han restado idoneidad, ya que las medidas de protección al ser decisiones que el Juez de Familia dicta para proteger de forma preventiva a la víctima de una posible agresión, en donde únicamente se verifica la situación de riesgo en la que se encuentra esta, y no se sanciona al supuesto agresor, estas se satisfacen por sí mismas, y por tanto, su vigencia no necesita estar supeditada a la sentencia emitida en el Juzgado Penal o 
hasta el pronunciamiento fiscal por el que se decida no presentar denuncia penal por resolución denegatoria, debiendo ser discreción del juez determinar el plazo de duración de estas medidas según las circunstancias particulares de cada caso en concreto. Por lo que, se concluye que las medidas de protección se encuentran inmersas en el concepto de medidas autosatisfactivas, que son soluciones jurisdiccionales urgentes, autónomas, y que importan una satisfacción definitiva de los requerimientos de los postulantes, no dependiendo su vigencia y mantenimiento de la interposición coetánea o ulterior de una pretensión principal.

Dicho problema, se ha puesto de manifiesto en el presente artículo, por lo que es necesario que las mismas sean superadas modificando la Ley 30364, específicamente el Art. 23, que regula la vigencia de las medidas de protección, ello con la finalidad de garantizar protección a las víctimas y crear un entorno seguro para todos los ciudadanos, ya que estamos viviendo en una sociedad donde el maltrato y la agresión forman parte del trato cotidiano de sus miembros, lo que acarreara un desequilibrio social que por su magnitud no podrá ser solucionado.

\section{BIBLIOGRAFÍA}

1. Alfaro, L. M. (2011). Delitos contra la familia y la violencia domestica. Lima: Jurista Editores.

2. Andia, J. R. (2008). Derecho de familia en el Codigo Civil. Lima: Demsa.
3. Anicama. Estudio epidemiologico sobre la violencia y comportamientos asociados en lima metropolitana y callao. Lima: Unfv.

4. Baptista, F. Y. (2014). Metodologia de la investigacion.

5. Bronferbrenner, U. (1987). La ecologia del desarrollo humano. Paidos.

6. Cabanellas De Torres, G. (2011). Diccionario Juridico Elemental. Heliasta.

7. Cabanellas, G. (2003). Diccionario Enciclopedico De Derecho Usual. Heliasta Argentina.

8. Canales, Y. G. (2011). Manual De Derecho De Familia. Lima: Jurista Editores.

9. Corsi, J. (2009). La Violencia a la Mujer en el contexto domestico. En Línea Http: //Www. Corsi. Com.Ar/Artículos. Htm.

10. Corsi, J. (2007). La Violencia de las mujeres como problema social.

11. Corsi, J. (1994). Una mirada abarcativa sobre el problema de la violencia familiar. Paidos.

12. Crhistian, S. B. (2009). Criminalizacion de la Violencia Familiar. Lima: Sociedad Juridica.

13. Diaz Pome, A. (2009). La efectividad de la Medidas De Proteccion Frente A La Violencia Familiar.

14. Humberto, N. A. (2014). Dignidad de la Persona, Derechos Fundamentales, Bloque Consti- 
tucional de Derechos y Control de Convencionalidad.

15. Iboamerica, X. C. (2008). 100 Reglas De Brasilia.

16. Martinez, A. Y. (1998). La Familia cedula fundamental de la sociedad. Yucatan Mexico: De La Comision De Derechos Humanos.

17. Molina, W. F. (2010). Violencia Familiar, Comentarios. Ediciones Legales.

18. Oms. (2013). Obtenido De https://es.wikipedia.org/ wiki/violencia_contra_la mujer\#cite_note-4

19. Oms. (2013). Estimaciones mundiales y regionales de la violencia contra la mujer: prevalencia y efectos de la violencia conyugal y de la violencia sexual no conyugal en la salud. Obtenido De http:// www.who.int/mediacentre/ news/releases/2013/violence_ against_women_20130620/es/

20. Oms. (2002). Informe mundial sobre la violencia y la salud. Obtenido De http://apps.who.int/ iris/bitstream/10665/67411/1/ a77102_spa.pdf
21. Pineda, J. (2008). Investigacion Juridica. Pacifico.

22. Ramos, O. M. (2010). Cifras de violencia familiar en el Peru. http://www. manuela.org.pe/ violencia.asp.

23. Reglamento De La Ley 30364 (2016).

24. Salas Beteta, C. (2009). Criminalizacion De La Violencia Familiar. Lima: Sociedad Juridica.

25. Sampieri, R. H. (2014). Metodologia de la Investigacion. Mc Graw Hill Education.

26. Sarlet, I. W. (2009). Dignidade Da Pessoa Humana E Direitos Fundamentais $\mathrm{Na}$ Constituicao Federal De 1988. Porto Alegre.

27. Universidad Externado, Colombia. (2011). Estrategias Metodológicas en la Investigación Sociojurídica. Bogotá: Xpress Estudio Gráfico Y Digital S.A.

28. Villanueva, R. (2003). Garantias Constitucionales y proteccion de los derechos de la Mujer. Lima: Revista De La Defensoria Del Pueblo. 
